Article

\title{
Factoring Continuous Homomorphisms Defined on Submonoids of Products of Topologized Monoids
}

\author{
Mikhail Tkachenko (D) \\ Department of Mathematics, Universidad Autónoma Metropolitana, Av. San Rafael Atlixco 186, Col. Vicentina, \\ Del. Iztapalapa, Mexico City C.P. 09340, Mexico; mich@xanum.uam.mx
}

Received: 13 June 2019; Accepted: 22 July 2019; Published: 26 July 2019

\begin{abstract}
We study factorization properties of continuous homomorphisms defined on submonoids of products of topologized monoids. We prove that if $S$ is an $\omega$-retractable submonoid of a product $D=\prod_{i \in I} D_{i}$ of topologized monoids and $f: S \rightarrow H$ is a continuous homomorphism to a topologized semigroup $H$ with $\psi(H) \leq \omega$, then one can find a countable subset $E$ of $I$ and a continuous homomorphism $g: p_{E}(S) \rightarrow H$ satisfying $f=g \circ p_{E}\left\lceil S\right.$, where $p_{E}$ is the projection of $D$ to $\prod_{i \in E} D_{i}$. The same conclusion is valid if $S$ contains the $\Sigma$-product $\Sigma D \subset D$. Furthermore, we show that in both cases, there exists the smallest by inclusion subset $E \subset I$ with the aforementioned properties.
\end{abstract}

Keywords: monoid; homomorphism; character; factorization

\section{Introduction}

The present article is a natural continuation of [1], where we study continuous mappings (of subspaces) of products of topological spaces and establish the existence of an irreducible factorization of those mappings under quite general assumptions. The survey article [2] by M. Hušek presents a well-structured exposition of the methods and results on factorization available prior to the year 1976.

Our purpose here is to consider the case when $f: S \rightarrow H$ is a continuous homomorphism of a submonoid $S$ of a product $D=\prod_{i \in I} D_{i}$ of topologized monoids. 'Topologized' means that the factors $D_{i}$ carry arbitrary topologies that can have no connection with multiplication on the monoids. For continuous homomorphisms of products of topological groups and subgroups of products, this study was initiated in [3-5].

As with [1], we are interested in identifying conditions under which a continuous homomorphism $f: S \rightarrow H$ admits an irreducible factorization in the form

$$
f=g \circ p_{J} \uparrow S
$$

where $J$ is a subset of the index set $I, p_{J}: D \rightarrow D_{J}=\prod_{i \in J} D_{i}$ is the projection, and $g: p_{J}(S) \rightarrow H$ is a continuous homomorphism. 'Irreducible' means that $J$ is the least by inclusion subset of $I$ for which $f$ can be decomposed as in (1). If one can find a finite (countable) set $J \subset I$ for which (1) holds true, we say that $f$ has finite (countable) type.

The very first result of this kind comes from the Pontryagin-van Kampen duality theory: Every continuous homomorphism of a product $D=\prod_{i \in I} D_{i}$ of compact abelian groups $D_{i}$ to the circle group $\mathbb{T}$ (called character) has finite type. S. Kaplan in [5] extends this result to products of reflexive (not necessarily locally compact) topological abelian groups.

In contrast, every product of infinitely many nontrivial (i.e., containing more than one point) Tychonoff spaces admits a continuous real-valued function which does not have finite type. The aforementioned facts show a big difference between the properties of continuous real-valued functions on the one hand, and continuous characters on the other. This difference is, indeed, 
even deeper. The following fact is purely algebraic: If $f$ is a homomorphism of a product $\prod_{i \in I} D_{i}$ of abelian groups to a slender group $H$, then $f$ has finite type provided the cardinality of the index set $I$ is less than the first uncountable Ulam measurable cardinal. [It is consistent with ZFC that such a cardinal does not exist.] An infinite cyclic group is the simplest example of a slender group (see [6]). The reader can consult [7] for more information on the algebraic component of this study.

Since the continuity of a homomorphism $f: G \rightarrow H$ of left topological groups is equivalent to the continuity of $f$ at the identity of $G$, one can expect that continuous homomorphisms defined on subgroups of products of left topological groups have countable type more frequently than continuous real-valued functions on products of spaces do. The following result proved in ([3], Lemma 8.5.4) confirms this conjecture and serves as a starting point for us:

Proposition 1. A continuous homomorphism $f: S \rightarrow H$ defined on an arbitrary subgroup $S$ of a product $D=\prod_{i \in I} D_{i}$ of left topological groups has countable type provided that the left topological group $H$ is a first countable $T_{1}$-space.

Several results on factorization of (weakly) continuous homomorphisms defined on subgroups of products of semitopological groups can be found in $[8,9]$.

In Section 2 we extend the validity of Proposition 1 to different situations, mainly when the factors $D_{i}$ and the codomain $H$ of $f$ are topologized monoids. These extensions of Proposition 1 are obtained at the cost of restricting the choice of a submonoid $S \subset D$. However, we weaken considerably the conditions on the factors $D_{i}$ and codomain $H$ of $f$. For example, in Corollaries 1 and 2 we show that Proposition 1 is valid for a submonoid $S$ of the product $D=\prod_{i \in I} D_{i}$ of topologized monoids $D_{i}$ provided that $S$ is either $\omega$-retractable (see Definition 1) or contains the $\Sigma$-product $\Sigma D \subset D$. Furthermore, we show that in both Corollary 1 and Corollary 2, there exists the least by inclusion subset $J$ of the index set $I$ such that the decomposition (1) is valid, i.e., $f$ admits an irreducible factorization. More general or complementing results are established in Theorem 1 and Proposition 2.

It is also worth noting that if the subgroup $S$ of $D$ in Proposition 1 satisfies either $S=D$ or $S=\Sigma D$, then one can weaken the requirement on $H$ by assuming that $H$ has countable pseudocharacter. This follows directly from Corollary 1 since both $D$ and $\Sigma D$ are retractable (see Definition 1).

By a product of a family $\left\{X_{i}: i \in I\right\}$ of topological spaces we always mean the topological product of this family, i.e., the Cartesian product set $X=\prod_{i \in I} X_{i}$ with the Tychonoff product topology, as defined in ([10], Section 2.3). This also applies in the case when the family $\left\{X_{i}: i \in I\right\}$ consists of (semi)topological (semi)groups or monoids.

\section{Notation and Preliminary Facts}

A semigroup is a nonempty set $S$ with a binary associative operation (called multiplication). A semigroup with an identity element is called a monoid. Clearly a monoid has a unique identity.

Assume that $G$ is a semigroup (monoid, group) with a topology. If the left shifts in $G$ are continuous, then $G$ is called left topological semigroup (monoid, group). If both left and right shifts in $G$ are continuous, then $G$ is said to be a semitopological semigroup (monoid, group). If multiplication in $G$ is jointly continuous, we say that $G$ is a topological semigroup. The concept of topological monoid is defined similarly. Furthermore, if $G$ is a group and multiplication in $G$ is jointly continuous, we say that $G$ is a paratopological group. A paratopological group with continuous inversion is a topological group.

The following simple fact is almost immediate from the above definitions (see e.g., [11], Chapter 1).

Lemma 1. Let $f: G \rightarrow H$ be a homomorphism of an abstract monoid (group) $G$ to a semitopological semigroup $H$. Then the image $f(G)$ with the topology inherited from $H$ is a semitopological monoid (group). Similarly, if $H$ is a topological semigroup, then $f(G)$ is a topological monoid (paratopological group).

One can easily extend Lemma 1 to the case when $H$ is a left or right topological semigroup. For example, if $f: G \rightarrow H$ is a homomorphism of an abstract monoid (group) $G$ to a left topological 
semigroup $H$, then the image $f(G)$ with the topology inherited from $H$ is a left topological monoid (group).

The next algebraic fact is well known and very easy to prove (see [11], Theorem 1.48).

Lemma 2. Let $f: D \rightarrow H$ and $p: D \rightarrow F$ be homomorphisms of semigroups such that the equality $p(x)=p(y)$ implies that $f(x)=f(y)$ whenever $x, y \in D$. If $p$ is surjective, then there exists a unique homomorphism $g: F \rightarrow H$ satisfying $f=g \circ p$.

Let $X=\prod_{i \in I} X_{i}$ be the product of a family $\left\{X_{i}: i \in I\right\}$ of spaces and $a=\left(a_{i}\right)_{i \in I} \in X$ be an arbitrary point. For every $x \in X$, we put

$$
\operatorname{diff}(x, a)=\left\{i \in I: x_{i} \neq a_{i}\right\}
$$

Then

$$
\Sigma X(a)=\{x \in X:|\operatorname{diff}(x, a)| \leq \omega\}
$$

is a dense subspace of $X$ called the $\Sigma$-product of the family $\left\{X_{i}: i \in I\right\}$ with center at $a$. If every factor $X_{i}$ is a monoid (group), we will always choose $a$ to be the identity $e$ of $X$. In the latter case, $\Sigma X(e)$ is a dense submonoid (subgroup) of the product $X$ and we abbreviate $\Sigma X(e)$ to $\Sigma X$.

Assume that $Z$ is a subset of the product $X=\prod_{i \in I} X_{i}$ of a family $\left\{X_{i}: i \in I\right\}$ of sets and $f: Z \rightarrow Y$ is an arbitrary mapping. We say that $f$ depends on $J$, for some $J \subset I$, if the equality $f(x)=f(y)$ holds for all $x, y \in Z$ with $p_{J}(x)=p_{J}(y)$, where $p_{J}: X \rightarrow \prod_{i \in J} X_{i}$ is the projection. It is clear that if $f$ depends on $J$, then there exists a mapping $g$ of $p_{J}(Z)$ to $Y$ satisfying $f=g \circ p_{J}\lceil Z$.

Definition 1. Let $D_{i}$ be a monoid with identity $e_{i}$, where $i \in I$. For a nonempty subset $J$ of $I$, we define a retraction $r_{J}$ of $D=\prod_{i \in I} D_{i}$ by letting

$$
r_{J}(x)_{i}= \begin{cases}x_{i} & \text { if } i \in J ; \\ e_{i} & \text { if } i \in I \backslash J,\end{cases}
$$

for each element $x \in D$. A subset $S$ of $D$ is said to be retractable if $r_{J}(S) \subset S$, for each $J \subset I$. If $\kappa$ is an infinite cardinal and the latter inclusion is valid for all subsets $J$ of I with $|J| \leq \kappa$, we say that $S$ is $\kappa$-retractable. Similarly, if the inclusion $r_{J}(S) \subset S$ holds for each finite set $J \subset I$, we call $S$ finitely retractable.

Clearly, the monoid $D=\prod_{i \in I} D_{i}$ is retractable, while $\Sigma D$ is a retractable submonoid of $D$. We use the notion of $\kappa$-retractability and its modifications in Theorem 1 and Proposition 2, meanwhile the retraction $r_{J}$ appears in the proofs of Lemmas 3 and 4.

Lemma 3. Let $D=\prod_{i \in I} D_{i}$ be the product of monoids $D_{i}$ and $e_{i}$ be the identity of $D_{i}$, where $i \in I$. Let also $S$ be a $\kappa$-retractable submonoid of $D$, for a cardinal $\kappa \geq \omega$. If $x, y \in S$ and $K, L$ are disjoint subsets of the index set $I$ with $|K| \leq \kappa$ and $|L| \leq \kappa$, then there exists an element $s \in S$ such that $p_{K}(s)=p_{K}(x), p_{L}(s)=p_{L}(y)$ and $s_{i}=e_{i}$ for each $i \in I \backslash(K \cup L)$.

Proof. Since $S$ is $\kappa$-retractable, $r_{K}(x)$ and $r_{L}(y)$ are in $S$. Then the element $s=r_{K}(x) \cdot r_{L}(y) \in S$ satisfies the equalities of the lemma.

Lemma 4. Let $Z$ be a subspace of the product space $X=\prod_{i \in I} X_{i}$ and $J$ be a nonempty subset of the index set $I$. If $r_{J}(Z) \subset Z$, then the restriction to $Z$ of the projection $p_{J}: X \rightarrow X_{J}=\prod_{i \in J} X_{i}$ is quotient when considered to be a mapping of $Z$ onto its image $p_{J}(Z) \subset X_{J}$.

Proof. The definition of $r_{J}$ implies that $p_{J}=p_{J} \circ r_{J}$. It is also clear that the restriction $p_{J} \uparrow r_{J}(X)$ is a homeomorphism between $r_{J}(X)$ and $p_{J}(X)=X_{J}$. Hence $p_{J} \uparrow r_{J}(Z)$ is a homeomorphism of $r_{J}(Z)$ 
onto $p_{J}(Z)$. The inclusion $r_{J}(Z) \subset Z$ means that $r_{J}\left\lceil Z\right.$ is a retraction of $Z$ onto its subspace $r_{J}(Z)$. Every retraction is a quotient mapping, so the mapping $p_{J}\lceil Z$ is quotient as the composition of the quotient mapping $r_{J}\left\lceil Z\right.$ and the homeomorphism $p_{J}\left\lceil r_{J}(Z)\right.$.

Given a space $X$, we denote by $P X$ the underlying set $X$ with the topology whose base consists of all nonempty $G_{\delta}$-sets in $X$. The space $P X$ is usually referred to as the $P$-modification of $X$. If $X$ is a (left) topological group or monoid, then $P X$ with the same multiplication is also a (left) topological group or monoid. If $P X=X$, i.e., every $G_{\delta}$-set in $X$ is open, we say that $X$ is a $P$-space.

Sometimes a product space $X=\prod_{i \in I} X_{i}$ is considered with the $\omega$-box topology whose base consists of the rectangular sets with countable supports,

$$
\left\{\prod_{i \in J} U_{i} \times \prod_{i \in I \backslash J} X_{i}: J \subset I,|J| \leq \omega, U_{i} \text { is open in } X_{i} \text { for each } i \in J\right\} .
$$

The $\omega$-box topology is always finer than the Tychonoff product topology.

The pseudocharacter of a space $X$ is the least cardinal $\kappa \geq \omega$ such that every point $x \in X$ is the intersection of at most $\kappa$ open sets in $X$. The pseudocharacter of $X$ is denoted by $\psi(X)$. Notice that the pseudocharacter is defined for $T_{1}$-spaces only.

\section{Factoring Continuous Homomorphisms}

First we introduce notation which is used all along this article.

Let $D=\prod_{i \in I} D_{i}$ be the product of a family $\left\{D_{i}: i \in I\right\}$ of monoids, $S$ be a submonoid of $D$, and $f: S \rightarrow H$ be a homomorphism to a semigroup $H$. Denote by $\mathcal{J}(f)$ the family of all sets $J \subset I$ such that $f$ depends on $J$. Our main concern is to determine the properties of the family $\mathcal{J}(f)$. For example, one can ask whether $\mathcal{J}(f)$ is a filter or whether it has a minimal (or even the smallest, by inclusion) element.

It turns out that the intersection of the family $\mathcal{J}(f)$, denoted by $J_{f}$, admits a clear description in terms of $f$. Let us say that an index $i \in I$ is $f$-essential if there exist points $x, y \in S$ such that $\operatorname{diff}(x, y)=\{i\}$ and $f(x) \neq f(y)$. Let $E_{f}$ be the set of all $f$-essential indices in $I$. According to ([1], Proposition 2.2), the equalities $E_{f}=\bigcap \mathcal{J}(f)=J_{f}$ are valid.

In the sequel a monoid (semigroup) with an arbitrary topology is called a topologized monoid (semigroup). In a topologized monoid, there can be no relation between multiplication and the topology of the monoid.

The following theorem is one of the main results of the article.

Theorem 1. Let $\kappa$ be an infinite cardinal, $S$ be a $\kappa$-retractable submonoid of a product $D=\prod_{i \in I} D_{i}$ of topologized monoids, and $f: S \rightarrow H$ a nontrivial continuous homomorphism to a topologized semigroup $H$ satisfying $\psi(H) \leq \kappa$. Then $E=\cap \mathcal{J}(f)$ is the smallest by inclusion element of the family $\mathcal{J}(f)$, $1 \leq|E| \leq \kappa$, and there exists a continuous homomorphism $g: p_{E}(S) \rightarrow H$ satisfying $f=g \circ p_{E} \mid S$, where $p_{E}: D \rightarrow \prod_{i \in E} D_{i}$ is the projection.

Proof. Clearly, the image $f(S)$ is a topologized monoid. Replacing $H$ with $f(S)$, if necessarily, we can assume that $H$ itself is a topologized monoid. Let $e$ and $e_{H}$ be the identity elements of $D$ and $H$, respectively. Notice that $H$ is a $T_{1}$-space since pseudocharacter is defined only for $T_{1}$-spaces.

Claim 1. There exists $J \in \mathcal{J}(f)$ with $|J| \leq \kappa$.

Indeed, it follows from $\psi\left(e_{H}, H\right) \leq \kappa$ and the continuity of $f$ that there exists a subset $J$ of $I$ with $|J| \leq \kappa$ such that $S \cap p_{J}^{-1} p_{J}(e) \subset f^{-1} f(e)=f^{-1}\left(e_{H}\right)$, where $p_{J}: D \rightarrow \prod_{i \in J} D_{i}$ is the projection. Let us verify that $f$ depends on $J$ and, hence, $J \in \mathcal{J}(f)$. 
Take arbitrary elements $x, y \in S$ such that $p_{J}(x)=p_{J}(y)$. Since $f$ is continuous and $\psi(H) \leq \kappa$, we can find a set $C \subset I$ such that $|C| \leq \kappa$ and $S \cap p_{C}^{-1} p_{C}(x) \subset f^{-1} f(x)$ and $S \cap p_{C}^{-1} p_{C}(y) \subset f^{-1} f(y)$. Clearly we can assume that $J \subset C$. Let $x^{\prime}=r_{C}(x)$ and $y^{\prime}=r_{C}(y)$. Then $x^{\prime}, y^{\prime} \in S$. Since $p_{C}\left(x^{\prime}\right)=p_{C}(x)$ and $p_{C}\left(y^{\prime}\right)=p_{C}(y)$, our choice of $C$ implies that $f\left(x^{\prime}\right)=f(x)$ and $f\left(y^{\prime}\right)=f(y)$.

Let $z=r_{J}(x), x^{\prime \prime}=r_{C \backslash J}(x)$ and $y^{\prime \prime}=r_{C \backslash J}(y)$. Then $\left\{z, x^{\prime \prime}, y^{\prime \prime}\right\} \subset S$ and $p_{J}\left(x^{\prime \prime}\right)=p_{J}\left(y^{\prime \prime}\right)=p_{J}(e)$. Hence $f\left(x^{\prime \prime}\right)=f\left(y^{\prime \prime}\right)=f(e)=e_{H}$, according to our choice of $J$. Notice that $x^{\prime}=x^{\prime \prime} \cdot z$ and $y^{\prime}=y^{\prime \prime} \cdot z$, which implies that $f\left(x^{\prime}\right)=f\left(x^{\prime \prime}\right) \cdot f(z)=f(z)$ and $f\left(y^{\prime}\right)=f\left(y^{\prime \prime}\right) \cdot f(z)=f(z)$. Therefore, we conclude that $f(x)=f\left(x^{\prime}\right)=f(z)=f\left(y^{\prime}\right)=f(y)$, which proves that $f$ depends on $J$ and $J \in \mathcal{J}(f)$. Claim 1 is proved.

Claim 2. If $x \in S$ and $E \cap \operatorname{diff}(x, e)=\varnothing$, then $f(x)=e_{H}$.

First we prove the claim in the special case when $\operatorname{diff}(x, e)$ is finite, say, $\operatorname{diff}(x, e)=\left\{i_{1}, \ldots, i_{n}\right\}$. Then can write $x=x_{1} \cdots x_{n}$, where $x_{1}, \ldots, x_{n}$ are elements of $S$ such that $\operatorname{diff}\left(x_{k}, e\right)=\left\{i_{k}\right\}$ for each $k=1, \ldots, n$ (here we use the finite retractability of $S$ ). By ([1], Proposition 2.2), the set $E=\cap \mathcal{J}(f)$ consists of all $f$-essential indices in $I$. Hence $f\left(x_{1}\right)=\cdots=f\left(x_{n}\right)=e_{H}$ because $i_{k} \notin E$ for each $k \leq n$. Therefore, $f(x)=f\left(x_{1}\right) \cdots f\left(x_{n}\right)=e_{H}$.

If $\operatorname{diff}(x, e)$ is infinite, we consider the set

$$
P=\left\{r_{F}(x): F \subset I \backslash E,|F|<\omega\right\} .
$$

Notice that $P \subset S$, by the finite retractability of $S$. It follows from the inclusion $\operatorname{diff}(x, e) \subset I \backslash E$ and our definition of $P$ that $x \in \bar{P}$, while $f(P)=\left\{e_{H}\right\}$ because the set $\operatorname{diff}(z, e)$ is finite and disjoint from $E$, for each $z \in P$. Since $f$ is continuous and takes values in the $T_{1}$-space $H$, we conclude that $f(x)=e_{H}$, as claimed.

Claim 3. $E \neq \varnothing$.

If $E$ is empty, then Claim 2 implies that $f(x)=e_{H}$ for each $x \in S$, which contradicts our assumption that $f$ is nontrivial.

Claim 4. $E=\cap \mathcal{J}(f)$ is the smallest by inclusion element of $\mathcal{J}(f)$.

Let us fix $J \in \mathcal{J}(f)$ as in Claim 1. Then $E \subset J$, so $|E| \leq|J| \leq \kappa$. It suffices to verify that $f$ depends on $E$. Consider arbitrary elements $x, y \in S$ with $p_{E}(x)=p_{E}(y)$. Since $|J| \leq \kappa$ and $S$ is $\kappa$-retractable, $x^{\prime}=r_{J}(x)$ and $y^{\prime}=r_{J}(y)$ are elements of $S$. If follows from the obvious equalities $p_{J}\left(x^{\prime}\right)=p_{J}(x)$ and $p_{J}\left(y^{\prime}\right)=p_{J}(y)$ and our choice of $J \in \mathcal{J}(f)$ that $f\left(x^{\prime}\right)=f(x)$ and $f\left(y^{\prime}\right)=f(y)$. Let $L=J \backslash E$. If $L=\varnothing$, then $E=J \in \mathcal{J}(f)$, as claimed. Thus, we can assume that $L \neq \varnothing$. Put $z=r_{E}\left(x^{\prime}\right), x^{\prime \prime}=p_{L}\left(x^{\prime}\right)$ and $y^{\prime \prime}=r_{L}\left(y^{\prime}\right)$. Then $\left\{z, x^{\prime \prime}, y^{\prime \prime}\right\} \subset S$ and the equality $p_{E}(x)=p_{E}(y)$ implies that $x^{\prime}=z \cdot x^{\prime \prime}$ and $y^{\prime}=z \cdot y^{\prime \prime}$. Notice that $\operatorname{diff}\left(x^{\prime \prime}, e\right)$ and $\operatorname{diff}\left(y^{\prime \prime}, e\right)$ are disjoint from $E$, so we can apply Claim 2 to conclude that $f\left(x^{\prime \prime}\right)=e_{H}$ and $f\left(y^{\prime \prime}\right)=e_{H}$. Therefore,

$$
f\left(x^{\prime}\right)=f\left(z \cdot x^{\prime \prime}\right)=f(z) \cdot f\left(x^{\prime \prime}\right)=f(z) \& f\left(y^{\prime}\right)=f\left(z \cdot y^{\prime \prime}\right)=f(z) \cdot f\left(y^{\prime \prime}\right)=f(z) .
$$

Summing up, we have that $f(x)=f\left(x^{\prime}\right)=f(z)=f\left(y^{\prime}\right)=f(y)$. Thus $f$ depends on $E$ and, hence, $E \in \mathcal{J}(f)$. This proves Claim 4 .

Since $f$ depends on $E$, Lemma 2 implies that there exists a homomorphism $g: p_{E}(S) \rightarrow H$ satisfying $f=g \circ p_{E}\left\lceil S\right.$. The continuity of $g$ follows from the fact that the restriction $p_{E}\lceil S$ is a quotient mapping (see Lemma 4). This completes the proof of the theorem.

We present below a special case of Theorem 1 for $\kappa=\omega$. Notice that Corollary 1 includes the case of the trivial homomorphism $f$, when $E=\varnothing$. 
Corollary 1. Let $D=\prod_{i \in I} D_{i}$ be a product of topologized monoids, $S$ be an $\omega$-retractable submonoid of $D$ and $f: S \rightarrow H$ a continuous homomorphism to a topologized semigroup $H$ of countable pseudocharacter. Then $E=\cap \mathcal{J}(f)$ is the smallest element of $\mathcal{J}(f)$ satisfying $|E| \leq \omega$ and $f$ has countable type.

According to Lemma 4, the retraction $r_{J}: S \rightarrow S$ of an $\omega$-retractable submonoid $S$ of $D$ is a quotient mapping, for each countable set $J \subset I$. We improve this result in the following lemma which is close to ([1], Lemma 1.3).

Lemma 5. Let $S$ be an $\omega$-retractable submonoid of the product $D=\prod_{i \in I} D_{i}$ of topologized monoids $D_{i}$. Then the restriction to $S$ of the projection $p_{J}: D \rightarrow D_{J}=\prod_{i \in J} D_{i}$ is an open homomorphism of $S$ onto its image $p_{J}(S)$, for each countable set $J \subset I$. The conclusion is valid for the three natural topologies on $D$ and $D_{J}$, the Tychonoff product topology, the $\omega$-box topology, and the P-modification of the latter.

Proof. We prove the lemma for the $\omega$-box topology on both $D$ and $D_{J}=\prod_{i \in J} D_{i} \supset p_{J}(S)$ - the arguments for the Tychonoff product topology and the $P$-modification of the $\omega$-box topology are almost the same.

Let $J$ be a nonempty countable subset of $I$. Take an arbitrary open set $U$ in $D$ such that $U \cap S \neq \varnothing$. We can assume without loss of generality that $U=p_{C}^{-1}(V)$ and $V=\prod_{i \in C} V_{i}$, where $C$ is a countable subset of $I$ and $V_{i}$ is open in $D_{i}$ for each $i \in C$. Clearly we can also assume that $J \subset C$. The conclusion of the lemma is immediate from the following claim:

Claim 5. $p_{J}(S \cap U)=p_{J}(S) \cap p_{J}(U)$.

The inclusion $p_{J}(S \cap U) \subset p_{J}(S) \cap p_{J}(U)$ is obvious. Let us verify that $p_{J}(S) \cap p_{J}(U) \subset p_{J}(S \cap U)$. Take an arbitrary point $z \in p_{J}(S) \cap p_{J}(U)$. Then $z=p_{J}(x)$ for some $x \in S$. Pick an element $t \in S \cap U$ and put $F=C \backslash J$. According to Lemma 3, there exists an element $s \in S$ such that $p_{J}(s)=p_{J}(x)$ and $p_{F}(s)=p_{F}(t)$. Since $s_{i}=x_{i}=z_{i} \in V_{i}$ for each $i \in J$ and $s_{i}=t_{i} \in V_{i}$ for each $i \in F$, we see that $s \in U$. Hence $s \in S \cap U$ and $z=p_{J}(x)=p_{J}(s) \in p_{J}(S \cap U)$. This proves the claim and the lemma.

Example 2.13 in [1] shows that Corollary 1 cannot be extended to arbitrary dense subgroups of products of topological groups, even if dense subgroups are pseudocompact.

The following lemma is evident; it shows that in the case of a continuous mapping $f$ defined on a $P$-space $X$, the codomain $Y$ of $f$ can be assumed discrete provided that $\psi(Y) \leq \omega$.

Lemma 6. Let $f: X \rightarrow Y$ be a continuous mapping, where $X$ is a P-space and $\psi(Y) \leq \omega$. Then $f$ remains continuous when $Y$ is endowed with the discrete topology.

Let $D=\prod_{i \in I} D_{i}$ be a product of topologized monoids and $P D$ be the $P$-modification of the space $D$. It turns out that a considerable part of Corollary 1 remains valid for continuous homomorphisms defined on certain submonoids of $P D$. This happens if the domain $S$ of a continuous homomorphism is either an $\omega$-retractable submonoid of $D$ or it contains the $\Sigma$-product $\Sigma D$. It is clear that the $P$-modification of $S$, denoted by $P S$, is a subspace (and submonoid) of $P D$.

First we present a well-known fact on restrictions of projections to 'big' dense subspaces of products (see e.g., [1], Lemma 2.10).

Lemma 7. Let $X=\prod_{i \in I} X_{i}$ be a product of spaces and $S$ be a subspace of $X$ such that $p_{J}(S)=X_{J}:=\prod_{i \in J} X_{i}$ for each countable set $J \subset I$, where $p_{J}: X \rightarrow X_{J}$ is the projection. Then the restriction $p_{J} \uparrow S$ is an open continuous mapping of $S$ onto $X_{J}$, for each $J \subset I$ with $|J| \leq \omega$.

Proposition 2. Let $D=\prod_{i \in I} D_{i}$ be a product of topologized monoids and $S$ be a submonoid of $D$ such that either 
(a) $\Sigma D \subset S$, or

(b) $S$ is $\omega$-retractable.

If $f$ is a continuous homomorphism of the submonoid PS of PD to a discrete semigroup $K$, then $f$ has countable type.

Proof. The image $f(S)$ is a submonoid of $K$. Let $e^{*}$ be the identity of $f(S)$. Since $K$ is discrete, the kernel of $f$ is an open submonoid of PS. Hence we can find a countable subset $J$ of the index set $I$ such that $S \cap p_{J}^{-1}\left(e_{J}\right) \subset \operatorname{ker} f$, where $e_{J}$ is the identity element of $D_{J}$. Denote by $e_{i}$ the identity of $D_{i}$, for each $i \in I$.

We claim that $f$ depends on $J$. The following argument is close to the proof of Claim 4 in Theorem 1 . Take arbitrary elements $x, y \in S$ such that $p_{J}(x)=p_{J}(y)$. Since $f$ is continuous and $K$ is discrete, we can find a countable set $C \subset I$ such that $S \cap p_{C}^{-1} p_{C}(x) \subset f^{-1} f(x)$ and $S \cap p_{C}^{-1} p_{C}(y) \subset f^{-1} f(y)$. Clearly we can assume that $J \subset C$. Let $x^{\prime}=r_{C}(x)$ and $y^{\prime}=r_{C}(y)$. Since $C$ is countable, $x^{\prime}, y^{\prime}$ are elements of $S$ in case (b), while $\left\{x^{\prime}, y^{\prime}\right\} \subset \Sigma D \subset S$ in case (a). It also follows from the equalities $p_{C}\left(x^{\prime}\right)=p_{C}(x)$ and $p_{C}\left(y^{\prime}\right)=p_{C}(y)$ and our choice of $C$ that $f\left(x^{\prime}\right)=f(x)$ and $f\left(y^{\prime}\right)=f(y)$.

Consider the elements $z=r_{J}(x)$ and $x^{\prime \prime}, y^{\prime \prime} \in D$ defined by $x_{i}^{\prime \prime}=e_{i}=y_{i}^{\prime \prime}$ for each $i \in J \cup(I \backslash C)$ and $x_{i}^{\prime \prime}=x_{i}, y_{i}^{\prime \prime}=y_{i}$ for each $i \in C \backslash J$. Again, $\left\{z, x^{\prime \prime}, y^{\prime \prime}\right\} \subset S$ in each of the cases (a) and (b). Notice that $p_{J}\left(x^{\prime \prime}\right)=p_{J}\left(y^{\prime \prime}\right)=e_{J}$, so our choice of $J$ implies that $f\left(x^{\prime \prime}\right)=f\left(y^{\prime \prime}\right)=e^{*}$. Also, we have that $x^{\prime}=x^{\prime \prime} \cdot z$ and $y^{\prime}=y^{\prime \prime} \cdot z$. The latter implies that $f\left(x^{\prime}\right)=f\left(x^{\prime \prime}\right) \cdot f(z)=f(z)$ and $f\left(y^{\prime}\right)=$ $f\left(y^{\prime \prime}\right) \cdot f(z)=f(z)$. Therefore, we conclude that $f(x)=f\left(x^{\prime}\right)=f(z)=f\left(y^{\prime}\right)=f(y)$, which proves the claim.

By virtue of Lemma 2, there exists a homomorphism $g$ of $p_{J}(S)$ to $K$ satisfying $f=g \circ p_{J} \uparrow S$. Since the projection $p_{J}: P D \rightarrow P D_{J}$ and its restriction to $P S$ are open mappings (we apply Lemma 7 in case (a) and Lemma 5 in case (b)), the homomorphism $g: p_{J}(S) \rightarrow K$ is continuous provided $p_{J}(S)$ carries the topology inherited from $P D_{J}$.

It is worth mentioning that (b) of Proposition 2 is a version of Corollary 1 for the P-modification of the product $D=\prod_{i \in I} D_{i}$. However, the family $\mathcal{J}(f)$ can fail to contain minimal elements under the conditions of Proposition 2, even if $f$ is a continuous character defined on the whole product $P D$. This follows from ([1], Example 2.16) if one replaces the unit interval $\mathbb{I}$ with the circle group $\mathbb{T}$ there.

An analogue of Proposition 2 for the Tychonoff product topology on $D$ is given below. It complements Corollary 1.

Corollary 2. Let $D=\prod_{i \in I} D_{i}$ be a product of topologized monoids and $S$ be a submonoid of $D$ such that $\Sigma D \subset S$. If $f$ is a continuous homomorphism of $S$ to a topologized semigroup $K$ with $\psi(K) \leq \omega$, then $E=\bigcap \mathcal{J}(f)$ is an element of $\mathcal{J}(f)$ satisfying $|E| \leq \omega$. Furthermore, $f$ has countable type.

Proof. Let $P D$ be the $P$-modification of the space $D$ and $P S$ be the submonoid $S$ endowed with the topology inherited from $P D$. Since $\psi(K) \leq \omega$, the space $P K$ is discrete. Hence, by Lemma 6 , the homomorphism $f: P S \rightarrow P K$ is continuous. According to Proposition 2, the homomorphism $f: P S \rightarrow P K$ has countable type. We can therefore find a countable set $J \subset I$ and a continuous homomorphism $g: p_{J}(S) \rightarrow P K$ satisfying $f=g \circ p_{J} \uparrow S$, where $p_{J}(S)$ carries the topology inherited from $P D_{J}$. We claim that $g$ remains continuous when considered as a homomorphism of $p_{J}(S)$ to $K$, where $p_{J}(S)$ inherits its topology from $D_{J}=\prod_{i \in J} D_{i}$.

Indeed, since $\Sigma D \subset S$, we have the equality $p_{A}(S)=D_{A}$, for each countable set $A \subset I$. Then the mapping $p_{J} \uparrow S$ of $S$ onto $D_{J}$ is open by Lemma 7 , whence the continuity of $g$ follows. Thus, $f$ has countable type.

Finally, making use of the inclusion $\Sigma D \subset S$, we can repeat the arguments in Claims 2-4 of the proof of Theorem 1 to conclude that $E=\cap \mathcal{J}(f)$ is an element of $\mathcal{J}(f)$. 
If the range $K$ of the homomorphism $f$ in Corollary 2 is a regular space, then one can weaken the condition imposed on the submonoid $S$ of $D$ as follows:

Corollary 3. Let $D=\prod_{i \in I} D_{i}$ be a product of topologized monoids and $S$ be a submonoid of $D$ such that $\sigma D \subset S$. If $f$ is a continuous homomorphism of $S$ to a topologized semigroup $K$ with $\psi(K) \leq \omega$ and the space $K$ is regular, then $E=\cap \mathcal{J}(f)$ is an element of $\mathcal{J}(f)$ satisfying $|E| \leq \omega$ and $f$ has countable type.

Proof. Since $K$ is regular it follows from ([1], Theorem 2.16) that the family $\mathcal{J}(f)$ contains the smallest element $E=\bigcap \mathcal{J}(f)$ and that the mapping $g: p_{E}(S) \rightarrow K$ satisfying $f=g \circ p_{E} \uparrow S$ is continuous. Therefore, to complete the proof, it suffices to show that $E$ is countable.

Let $f^{*}$ be the restriction of $f$ to $\sigma D$. Since $\sigma D \subset S$ and $K$ is regular (hence Hausdorff), we can apply ([1], Lemma 2.15) to obtain the equality $E=\bigcap \mathcal{J}(f)=\bigcap \mathcal{J}\left(f^{*}\right)$. Clearly $\sigma D$ is a retractable submonoid of $D$ and $f^{*}$ is a continuous homomorphism of $P(\sigma D)$ to the discrete semigroup $P K$. Therefore, by (b) of Proposition 2, $f^{*}$ has countable type (notice that the definition of $\mathcal{J}(f)$ does not depend on the topologies of $S$ and $K)$. Hence the family $\mathcal{J}\left(f^{*}\right)$ contains a countable element, say, $C$. Since $E=\bigcap \mathcal{J}\left(f^{*}\right) \subset C$, the set $E$ is countable as well. In particular, $f$ has countable type.

Our next aim is to present Theorem 2 whose proof requires three preliminary facts. The first and second of them are close to Theorem 1.7.2 and Proposition 1.6.22 of [3], respectively, while the third one is almost evident.

A space $X$ is called pseudo- $\omega_{1}$-compact if every locally finite family of open sets in $X$ is countable. Several authors use the term discrete countable chain condition (DCCC, for brevity) in place of pseudo- $\omega_{1}$-compactness. The following proposition is a special case of ([12], Theorem 5).

Proposition 3. Let $S$ be a dense subspace of a product space $X=\prod_{i \in I} X_{i}$ and $f: S \rightarrow Y$ be a continuous mapping to a space $Y$ with a regular $G_{\delta}$-diagonal. If $S$ is pseudo- $\omega_{1}$-compact, then there exists a countable set $J \subset I$ such that $f$ depends on $J$.

The nontrivial part of the next lemma was announced in [13] as Lemma 2 and proved in a more general form in [14].

Lemma 8. Let $S$ be a subspace of a product $X=\prod_{i \in I} X_{i}$ of spaces such that $p_{J}(S)=\prod_{i \in J} X_{i}$, for each finite set $J \subset I$. Then $X$ is pseudo- $\omega_{1}$-compact if and only if so is $S$.

A subset $Z$ of a space $X$ is said to be a zero-set if there exists a continuous real-valued function $f$ on $X$ such that $Z=f^{-1}(0)$. The diagonal of $X$ is the subset $\Delta_{X}=\{(x, x): x \in X\}$ of $X \times X$. Hence $X$ has a zero-set diagonal if $\Delta_{X}$ is a zero-set in $X \times X$. Notice that every zero-set is the intersection of countably many closed neighborhoods. This implies that a space with a zero-set diagonal has a regular $G_{\delta}$-diagonal. The next fact is known in folklore and can be proved in several distinct ways. We present a direct argument with the use of prenorms on topological groups (see [3], Section 3.3).

Lemma 9. Every topological group G of countable pseudocharacter has a zero-set diagonal.

Proof. Since $\psi(G) \leq \omega$, there exists a sequence $\left\{U_{n}: n \in \omega\right\}$ of open symmetric neighborhoods of the identity $e$ in $G$ such that $U_{n+1}^{2} \subset U_{n}$ for each $n \in \omega$ and $\{e\}=\bigcap_{n \in \omega} U_{n}$. It follows from ([3], Lemma 3.3.10) that there exists a continuous prenorm $N$ on $G$ satisfying

$$
\left\{z \in G: N(z)<1 / 2^{n}\right\} \subset U_{n}
$$

for each $n \in \omega$. We define a continuous real-valued function $h$ on $G \times G$ by letting $h(x, y)=N\left(x^{-1} y\right)$, for all $x, y \in G$. It remains to verify that $\Delta_{G}=h^{-1}(0)$. Clearly $h(x, x)=0$ for each $x \in G$. Take arbitrary elements $x, y \in G$ with $x \neq y$. Then $z=x^{-1} y \neq e$, so $z \notin U_{n}$ for some $n \in \omega$. Hence our choice of $N$ 
and the definition of $h$ imply that $h(x, y)=N(z) \geq 1 / 2^{n}>0$. This proves the equality $\Delta_{G}=h^{-1}(0)$ and shows that $\Delta_{G}$ is a zero-set in $G \times G$.

The following result is of almost pure topological character; we think nevertheless that it presents some interest in the context of topological algebra.

Theorem 2. Let $S$ be a subspace of a product $D=\prod_{i \in I} D_{i}$ of topological spaces such that $p_{J}(S)=\prod_{i \in J} D_{i}$, for each countable set $J \subset$ I. If $D$ is pseudo- $\omega_{1}$-compact, then every continuous mapping $f: S \rightarrow K$ to a topological group $K$ with $\psi(K) \leq \omega$ has countable type. The same conclusion is valid if $K$ is a Hausdorff paratopological group of countable $\pi$-character.

Proof. If $K$ is a topological group with $\psi(K) \leq \omega$, then Lemma 9 implies that $K$ has a regular $G_{\delta}$-diagonal. Similarly, if $K$ is a Hausdorff paratopological group of countable $\pi$-character, then we use ([15], Theorem 25) to conclude that $K$ has a regular $G_{\delta}$-diagonal.

Applying Lemma 8 we see that $S$ is pseudo- $\omega_{1}$-compact, so Proposition 3 implies that there exists a countable set $J \subset I$ such that $f$ depends on $J$. Hence we can find a mapping $g$ of $p_{J}(S)=\prod_{i \in J} D_{i}$ to $K$ satisfying $f=g \circ p_{J} \backslash S$. It follows from our assumptions about $S$ that the restriction of $p_{J}$ to $S$ is an open mapping (see Lemma 7), so the equality $f=g \circ p_{J}\lceil S$ implies that $g$ is continuous. Thus, $f$ has countable type.

Our last example (which we borrow from [1]) shows, in particular, that the condition $\Sigma D \subset S$ on the submonoid $S$ of $P D$ in (a) of Proposition 2 or in Corollary 2 cannot be weakened to the density of $S$ in $D$, nor even in $P D$.

Let us recall that a character of an abstract group $G$ is a homomorphism of $G$ to the torus $\mathbb{T}$. Since the discrete group $\mathbb{Z}(2)=\{-1,1\}$ is a subgroup of $\mathbb{T}$, every homomorphism of a group $G$ to $\mathbb{Z}(2)$ is a character. We denote the power of the continuum by $\mathfrak{c}$, so $\mathfrak{c}=2^{\omega}$.

Example 1. (See [1], Example 2.13) Let $p$ be the projection of the compact topological group $\Pi=\mathbb{Z}(2)^{\mathfrak{c}} \times \mathbb{Z}(2)$ to the second factor $\mathbb{Z}(2)$. There exists a dense pseudocompact subgroup $S$ of $\Pi$ such that $\chi=p\left\lceil S\right.$ depends on each of the factors $\mathbb{Z}(2)^{\mathfrak{c}}$ and $\mathbb{Z}(2)$. In particular, $\chi$ is a continuous character of a dense subgroup of $\Pi$, but the family $\mathcal{J}(\chi)$ fails to be a filter and has no smallest element.

The forthcoming article [16] continues this line of our study but it focuses more on the cases where a continuous homomorphism $f: S \rightarrow K$ depends on a finite subset of the index set $I$.

All main results in Section 2, except for Proposition 3 and Theorem 2, concern continuous homomorphism defined on submonoids of products of topologized monoids. This gives rise to the following general problem:

Problem 1. Which of the results in Section 2 remain valid for continuous homomorphisms defined on subsemigroups of products of topological semigroups?

Needless to say, the existence of the identity element in monoids has been crucial in the major part of our arguments here.

Funding: This research received no external funding.

Acknowledgments: The author is grateful to the referees for constructive comments and useful remarks and, especially, for providing the references [12,14].

Conflicts of Interest: The author declares no conflict of interest.

\section{References}

1. Tkachenko, M. Factoring continuous mappings defined on subspaces of topological products. Topol. Appl. 2019, accepted. 
2. Hušek, M. Continuous mappings on subspaces of products. Symp. Math. 1976, 17, $25-41$.

3. Arhangel'skii, A.V.; Tkachenko, M.G. Topological Groups and Related Structures; van Mill, J., Ed.; Atlantis Press: Paris, France; Amsterdam, The Netherlands, 2008.

4. Hušek, M. Productivity of properties of topological groups. Topol. Appl. 1992, 44, 189-196. [CrossRef]

5. Kaplan, S. Extension of the Pontrjagin duality I: Infinite products. Duke Math. J. 1948, 15 649-658. [CrossRef]

6. Nunke, R.J. Slender groups. Bull. Am. Math. Soc. 1961, 67, 274-275. [CrossRef]

7. Bergman, G.M. Homomorphisms on infinite direct products of groups, rings and monoids. Pac. J. Math. 2015, 274, 451-495. [CrossRef]

8. Batíková, B.; Hušek, M. Productivity of Coreflective Subcategories of Semitopological Groups. Appl. Categor. Struct. 2016, 24, 497-508. [CrossRef]

9. Hušek, M.; Rosický, J. Factorization and local presentability in topological and uniform spaces. Topol. Appl. 2019, 259, 251-266. [CrossRef]

10. Engelking, R. General Topology; Heldermann Verlag: Berlin, Germany, 1989.

11. Carruth, J.H.; Hildebrant, J.A.; Koch, R.J. The Theory of Topological Semigroups; Marcel Dekker, Inc.: New York, NY, USA; Basel, Switzerland, 1983; Volume I.

12. Husek, M. Mappings from products. Topol. Struct. II 1979, 115, 131-145.

13. Comfort, W.W.; Negrepontis, S. Extending continuous functions on subspaces of product spaces-Preliminary report. In Notices of the American Mathematical Society; American Mathematical Society: Providence, RI, USA, 1971; Volume 18, p. 669.

14. Comfort, W.W.; Negrepontis, S. Continuous functions on products with strong topologies. In Proceedings of the Third Prague Topological Symposium 1971, Prague, Czech Republic, 30 August-3 September 1971; Academia Publishing House of the Czechoslovak Academy of Sciences: Praha, Czech Republic, 1972; pp. 89-92.

15. Sánchez, I. Cardinal invariants of paratopological groups. [CrossRef] Topol. Algebra Appl. 2013, 1, 37-45.

16. Tkachenko, M. Continuous Homomorphisms Defined on (Dense) Submonoids of Products of Topologized Monoids. Preprint.

(C) 2019 by the author. Licensee MDPI, Basel, Switzerland. This article is an open access article distributed under the terms and conditions of the Creative Commons Attribution (CC BY) license (http:/ / creativecommons.org/licenses/by/4.0/). 